\title{
Load Balancing Routing Algorithm among Multiple Gateways in MANET with Internet Connectivity
}

\author{
Yonghang Yan*, Linlin Ci*, Ruiping Zhang**, Zhiming Wang* \\ *School of Computer Science, Beijing Institute of Technology, Beijing, China \\ **School of Computer Science, Zhongyuan University of Technology, Zhengzhou, China \\ feifanyyh@gmail.com, cilinlin@263.net, zrpwhut@163.com,bj_wzm@sohu.com
}

\begin{abstract}
When MANET is connected into the Internet, the gateways are the entry points of all traffic between the hybrid networks. Usually, a large amount of traffic load may become concentrated in some gateways and congestion occurs. To resolve this problem, this paper proposes a load balancing routing algorithm, called LBRA, in MANET to distribute the traffic load among multiple gateways. Through simulation, we show that the proposed algorithm can distribute the traffic load uniformly among multiple gateways and suppress the increase in the routing path length at most $20 \%$ compared to the shortest path routing.
\end{abstract}

Keywords — Load balancing; multiple gateways; routing; MANET; Internet connectivity

\section{INTRODUCTION}

A mobile ad hoc network (MANET) [1] is a wireless interconnection of nodes that provides mobility and communication between nodes without a central entity like a base station or an access point. This allows the mobile nodes to setup cost effective networks quickly and without any fixed topology. Originally meant for use in military applications, MANET can also play an important role in relief and rescue operations as well as in various other personal area networking applications such as team meeting.

In the development of MANET, we have observed that most of the existing works have assumed a stand-alone MANET. If one can simultaneously enjoy both the flexibility provided by MANET and the tremendous services and data provided by the Internet, it would be more attractive. So, connecting MANET into the Internet is becoming more and more popular. As we all know, there are huge differences between MANET and the Internet, so a special device which acts as a "bridge" between these two heterogeneous networks is needed. This special device is called a gateway in this paper. All the packets from/to the Internet are routed by the gateway.

In order to enhance the reliability and robustness of the interconnection between MANET and the Internet, multiple gateways are usually deployed in these hybrid networks. A key issue in such a hybrid network is how to utilize the bandwidths provided by the gateways efficiently. There are several concerns to be addressed in this issue. First, the traffic concentration effect, which is due to multiple mobile hosts connecting a special gateway, can easily saturate this gateway.
Second, considering node mobility, the members of a gateway (and thus the aggregated traffic load) may change dynamically. Third, gateways may be heterogeneous, which further increases the difficulty of this problem.

To solve this issue, some load balancing routing approaches among multiple gateways are proposed ([2]-[6]). Note that the various problems of existing schemes, a novel load balancing routing algorithm among multiple gateways is presented in this paper. This algorithm can efficiently distribute the traffic load evenly among different gateways and maximize the interconnection throughput between MANET and the Internet. Reference [7] shows that throughput performance of wireless multi-hop session is seriously degraded with increase of the number of hops. When our algorithm calculates a load balanced path to a far gateway, throughput performance may yet not improved. In extreme case of selecting far further gateway, throughput performance through gateway may be degraded seriously and our load balancing routing algorithm may bring worse throughput. In order to control the length increment of the routing path for realizing the load balancing among gateways, a threshold of length increment of routing path is introduced to avoid consuming more wireless resources and prevent the throughput degradation.

The remaining of this paper is organized as follows. In the next section, we describe the system model. The proposed load balancing routing algorithm is presented in section III. Section IV presents various simulation results to verify the effectiveness of the proposed algorithm. Some conclusions are given in Section $\mathrm{V}$.

\section{SYSTEM MODEL}

In this section, we first define the system model of an interconnection between MANET and the Internet and an example is shown in Figure 1. The load balancing routing problem is researched under such an environment. We assume that the interconnection architecture is mainly used to support Internet access. A mobile node without a direct Internet link can access the Internet through the gateway. Therefore, the service area of the Internet is extended by the ad hoc links.

From the system model, we can achieve the interconnection network model. We assume a communication graph $G=(V$, $E$ ), where $V=\left\{v_{l}, \ldots, v_{m}, \ldots, v_{n}\right\}$ is the set of mobile nodes ( $n$ $\geq m \geq 1, n$ is the number of mobile nodes, $m$ is the number of 
gateways, and mobile nodes from $v_{l}$ to $v_{m}$ are gateways), and $E$ is the set of links $l_{i, j}=\left(v_{i}, v_{j}\right)$ if they have direct link. To solve the load balancing routing problem, we model the interconnection network architecture by a node-weighted graph as follows. Each mobile node $v_{i}$ is translated into a pair $\left(v_{i}, T_{i}\right)$, where $T_{i}$ is the traffic load of $v_{i}$, and it represents the traffic load between $v_{i}$ and the Internet. Note that we mainly consider the interconnection architecture as an Internet access environment, and therefore do not consider inter-node (mobile node) traffics. For each gateway $v_{j}(m \geq j \geq 1)$ with the capacity of $C_{j}$, it is translated into $\left(v_{j}, C_{j}\right)$. An example is shown in Figure 2.

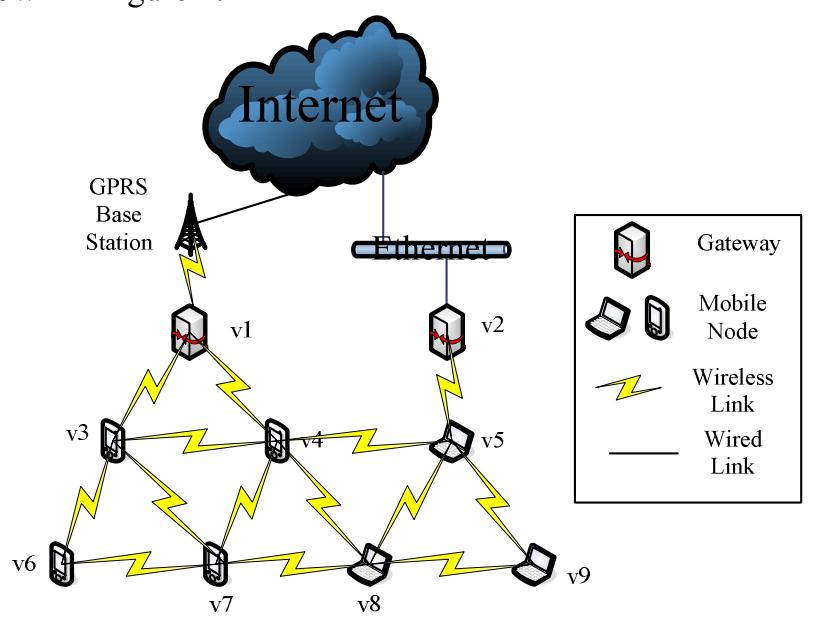

Figure 1. An example of the interconnection between MANET and the Internet.

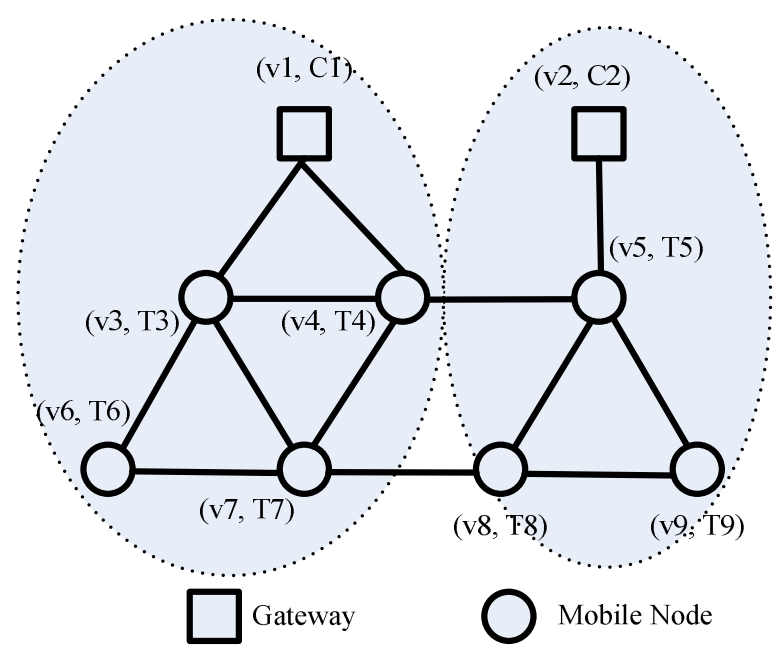

Figure 2. The graph model of the interconnection architecture between MANET and the Internet in Figure 1.

In Figure 2, gateways and mobile nodes are represented by squares and circles, respectively. Gateways are sinks in the network, while the other mobile nodes' traffic loads need to be drained out to proper sinks with considering load balance. One possible solution is shown in Figure 2, where mobile nodes are divided, based on shortest path routing algorithm, into two groups of sizes 4 and 3 to be served by gateways $v_{1}$ and $v_{2}$, respectively. Considering the gateways having different capacities, such arrangement is obviously imbalanced in most of the cases. If we assume the capacity of gateways $v_{1}$ and $v_{2}$ are $256 \mathrm{kbps}$ and $11 \mathrm{Mbps}$, respectively, then gateway $v_{2}$, which has the largest bandwidth, is responsible for 3 mobile nodes. So it is possible that gateway $v_{l}$ may run out all its bandwidth, while gateway $v_{2}$ still has unused bandwidth.

\section{Routing ALgORITHM}

In this section, we first define all gateways load balance factor $(G L B F)$ and then present the load balancing routing algorithm among multiple gateways.

\section{A. Calculating All Gateways Load Balance Factor}

To make the mobile nodes find out an optimal routing path to the gateway to distribute the traffic load evenly among multiple gateways, we define the concept of all gateways load balance factor $(G L B F)$ as follows. Without loss of generality, we assume that the network is connected. Otherwise, either some mobile nodes are not connected to any gateway, or the problem can be divided into some sub-problems which can be resolved independently.

Let $v_{j}(m \geq j \geq 1)$ be any gateway node. Let $S_{j}$ be the set of mobile nodes which are connected to the gateway $v_{j}$. Then the remaining available bandwidth of gateway $v_{j}$, denoted by $\theta_{j}$, is defined to be $C_{j}$ subtracted by the sum of the traffic load from the mobile nodes which are connected to this gateway, as is shown in (1).

$$
\theta_{j}=\max \left\{\left(C_{j}-\sum_{i \in S_{j}} T_{i}\right), 0\right\}
$$

The $\max$ function is to ensure that $\theta_{j}$ is always non-negative. The load factor $L F_{j}$ of gateway $v_{j}$ is defined by (2).

$$
L F_{j}=\frac{\sum_{i \in S_{j}} T_{i}}{C_{j}}
$$

Note that the value of $L F_{j}$ could be greater than 1 , in case that the gateway is overloaded. Then we can define all gateways load balance factor $G L B F$, as is shown in (3).

$$
G L B F=\left\{\begin{array}{ccc}
\frac{\max \left\{\theta_{j}\right\}-\min \left\{\theta_{j}\right\}}{\max \left\{\theta_{j}\right\}} & \text { if } & \max \left\{\theta_{j}\right\}>0 \\
\frac{\max \left\{L F_{j}\right\}-\min \left\{L F_{j}\right\}}{\max \left\{L F_{j}\right\}} & \text { if } & \max \left\{\theta_{j}\right\}=0
\end{array}\right.
$$

In equation $(3), j(m \geq j \geq 1)$ represents all the gateways. The former is applied when at least one gateway has remaining available bandwidth, while the latter is applied when all the gateways are fully loaded. In both cases, a load balancing routing scheme's goal is to minimize the GLBF value possibly.

\section{B. Load Balancing Routing Algorithm Among Multiple Gateways}


In the process of calculating the routing path to the gateway to achieve load balancing among multiple gateways, the length of the routing path calculated by our algorithm may be very long compared to the one calculated by the shortest path routing algorithm. Reference [7] shows that throughput performance of wireless multi-hop session is seriously degraded with increase of the number of hops. So, when our algorithm calculates a routing path to a further gateway, throughput performance may yet not be improved. In extreme case of finding out a routing path to a far further gateway, throughput performance through this gateway may be degraded seriously and our algorithm may bring worse throughput. So, we use the shortest path routing algorithm as a baseline algorithm and introduce a routing path increment threshold to restrict the length of routing path calculated by our algorithm in a reasonable range.

Table 1 shows the load balancing routing algorithm among multiple gateways (LBRA). We show the overview of this algorithm as follows.

- STEP 1: For all $v_{i}(n \geq i \geq m+1)$, calculate the shortest path from $v_{i}$ to its nearest gateway using shortest path routing algorithm. Store $v_{i}$ in $V_{s}$, record the routing path in $P_{i, g i}$ and compute its length in vector length . $_{\text {. }}$ Compute the remaining available bandwidth of each gateway using equation (1) and gateway load factor using equation (2). Then compute all gateways load balance factor stored by $L B F_{0}$ using equation (3).

- STEP 2: For all $v_{i} \in V_{s}$, repeat the following steps.

- STEP 3: Find all $v_{j}$ such that $l_{i, j} \in E$ and push $v_{j}$ into Stack.

- STEP 4: If the Stack is empty, then returns to STEP 2. Otherwise, pop up the top element of the stack and assign this element to $v_{k}$. If $k \leq m$, then go to STEP 4-1. Otherwise, go to STEP 4-2.

- STEP 4-1: Save the current value of the gateway $g_{i}$ and $g_{k}$. Recalculate the remaining available capacity of the gateway $g_{i}$ and $g_{k}$ and the all gateways load balance factor stored by $L B F_{k}$. Then determine whether the following two conditions are satisfied at the same time: (1) $L B F_{k}<L B F_{0}$, (2) $1+\left|P_{k g k}\right| \leq$ Length $_{i}+h_{\text {thres. }}$. If they are satisfied, then replace $P_{i, g i}$ with $\left\{l_{i, k}\right\} \cup P_{k g k}$ and assign $L B F_{k}$ to $L B F_{0}$, go to STEP 4. Otherwise, restore the value of the gateway $g_{i}$ and $g_{k}$ and go to STEP 4 .

- STEP 4-2: Find all the link $l_{k, l} \in E$ and push $v_{l}$ into the Stack. Then go to STEP 4.

LBRA exhaustively searches for all the possible routing paths from $v_{i}$ to $v_{g i}$. So, the worst-case time complexity of this algorithm occurs with a full connected network topology. In this case, each mobile node has $m$ links between the gateways and $(n-m-1)$ links between the other mobile nodes. Therefore, $(n-m)$ mobile nodes search for $((n-m) m+(n-m-1) !)$ paths. The shortest path routing (SPR) uses Dijkstra's algorithm [8] for calculating the shortest path, the time complexity of obtaining the path between a mobile node and the gateway is $O\left(n^{2}\right)$. In $\mathrm{SPR}$, each mobile node repeats this operation $m$ times. Then it determines the path to the gateway within $O(n)$. So, the time complexity of SRP is $O\left(n^{2}\right)$. Then the time complexity of LBRA is the sum of $((n-m) m+(n-m-1) !)$ and $O\left(n^{2}\right)$. In the end, the time complexity of LBRA is $O((n-m) !)$.

TABLE 1. LOAD BALANCING ROUTING ALGORITHM AMONG MULTIPLE GATEWAYS

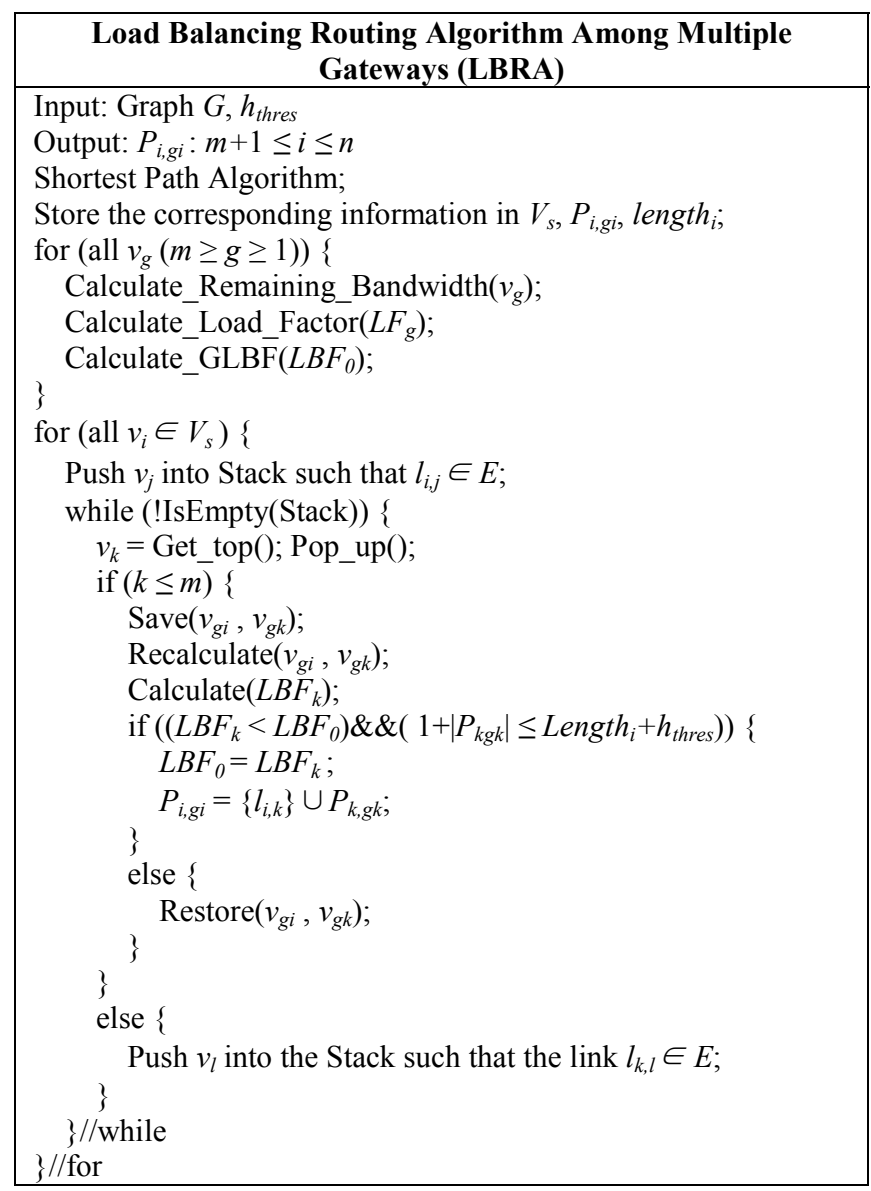

\section{Performance Evaluation}

In this section, through simulation using ns-2.34 network simulator [9] with CMU wireless extensions, we demonstrate the effectiveness of the proposed algorithm in terms of the load on the gateways and the path length.

\section{A. Simulation Setup}

The simulation area is $1200 \mathrm{~m} * 1200 \mathrm{~m}$. The node mobility speed we chosen in the simulation varies from 10 to $20 \mathrm{~m} / \mathrm{s}$. The random waypoint model is used as the mobility model of the mobile nodes. Constant Bit Rate (CBR) traffic sources with different packet generation rates are used to model different network scenarios. The maximum transmission range of each node is 250 meters. Simulations are run for $600 \mathrm{~s}$.

In addition, the gateways are deployed as follows. For 2 gateways, they are deployed in the diagonal of the square. For 4 gateways, they are deployed in the vertex of the square. For 8 gateways, they are deployed in the vertex of the square and in the midpoint of the edge of the square. This is because the numbers of gateway nodes and their locations are carefully 
determined to maximize the throughput between MANET and the Internet. The simulation parameters are given according to Table 2 .

TABLE 2. SIMULATION PARAMETER

\begin{tabular}{|l|l|}
\hline \multicolumn{1}{|c|}{ Parameter } & \multicolumn{1}{c|}{ Value } \\
\hline Simulation Area & $1200 \mathrm{~m} \times 1200 \mathrm{~m}$ \\
\hline Wireless MAC Interface & IEEE $802.11 \mathrm{MAC}$ \\
\hline Propagation Model & Two Ray Ground \\
\hline Transmission Range & $250 \mathrm{~m}$ \\
\hline Gateway Nodes & $2,4,8$ \\
\hline CBR Packet Size & 512 bytes \\
\hline Mobility Model & Random Waypoint \\
\hline MANET node speed & $10-20 \mathrm{~m} / \mathrm{s}$ \\
\hline Simulation Time & $600 \mathrm{~s}$ \\
\hline
\end{tabular}

\section{B. Optimal Path Increment Threshold Setting}

Under the premise of distributing traffic load evenly among multiple gateways, LBRA suppresses the increase in the hop count caused by adjusting the routing path up to $h_{\text {thres }}$. The optimal routing path increment threshold is investigated from the viewpoints of both the load on the gateways and the path length.

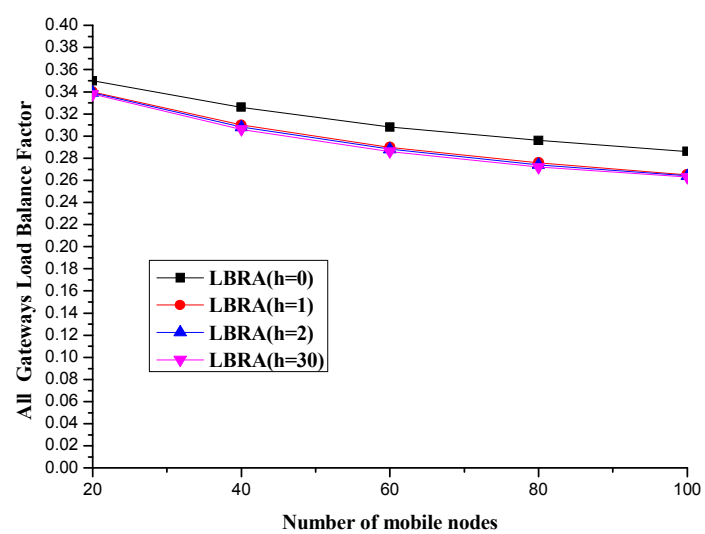

Figure 3. Relationship between $h_{\text {thres }}$ and all gateways load balance factor.

Figure 3 illustrates the relationship between $h_{\text {thres }}$ and all gateways load balance factor when the number of mobile nodes varies and the number of gateways is set to 4 . As shown in Figure 3, the all gateways load balance factor does not change if $h_{\text {thres }}$ is over 1 . The case that $h_{\text {thres }}$ equals to 30 represents the routing path increment threshold is unlimited. Because of obtaining similar results in other cases with different numbers of gateways, we set $h_{\text {thres }}=1$ in the following evaluation.

\section{Load Balance on Gateways}

We deploy 2, 4 and 8 gateways respectively in the connected hybrid network to investigate the impact of SPR and our LBRA algorithm on the load balance on gateways when the number of mobile nodes varies. The results are shown in Figure 4. As earlier noted, the locations of the gateways are carefully determined to maximize the throughput between MANET and the Internet. From Figure 4, we can see that the all gateways load balance factor of the two algorithms become low as the number of mobile nodes increases. The degree of reducing the all gateways load balance factor of LBRA is more obvious. The LBRA can distribute the load among gateways more uniformly.

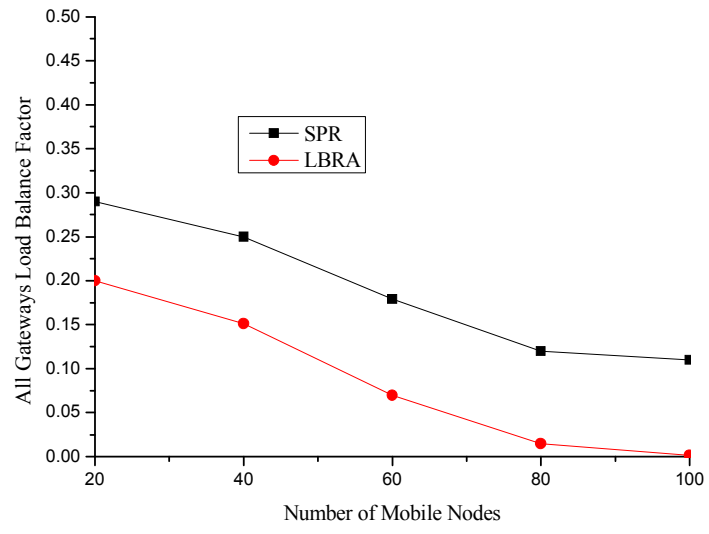

a. 2 gateways
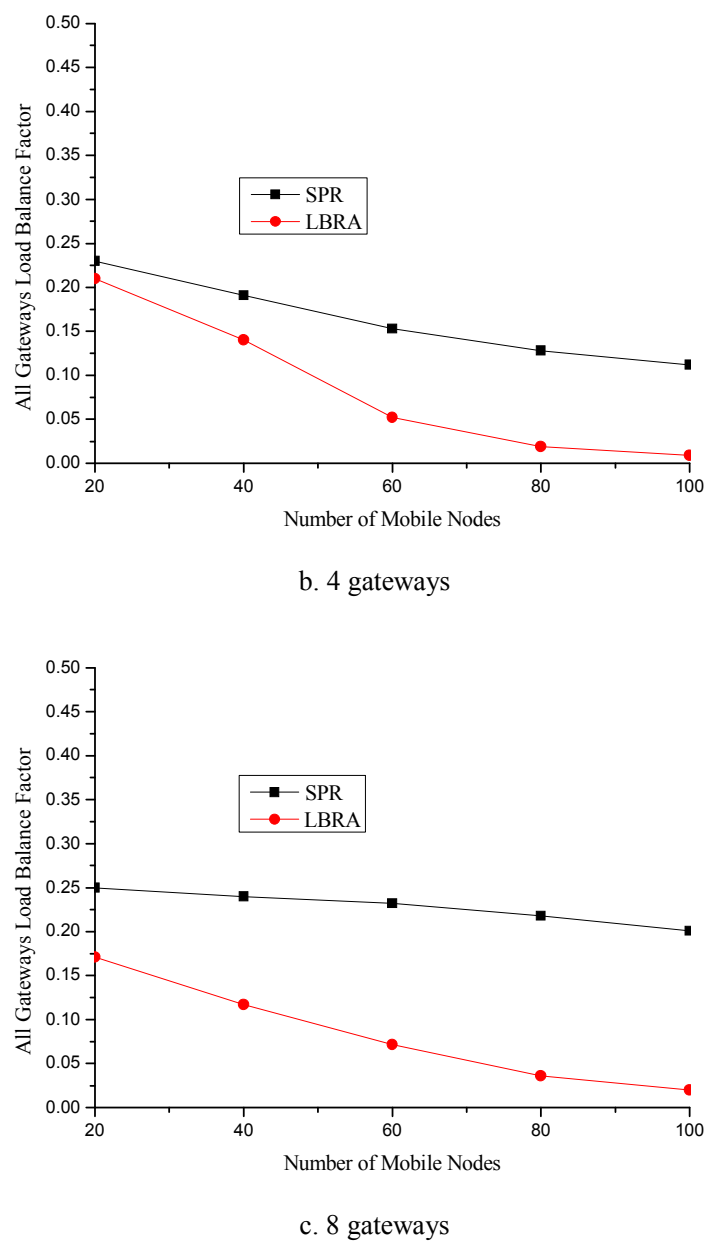

Figure 4. All gateways load balance factor changes with the increment of the number of mobile nodes with 2, 4 and 8 gateways respectively. 


\section{The Impact on Average Routing Path Length}

Figure 5 depicts how the average routing path length between a mobile node and a gateway varies according to the number of gateways when the number of mobile nodes is 40 . Figure 6 shows how the average routing path length between a mobile node and a gateway varies according to the number of mobile nodes when the number of gateways is 4 . We find the average routing path length of LBRA is at most $20 \%$ higher than that of SPR. So, when using our LBRA to distribute the load evenly among multiple gateways, the increase of the routing path length is not large. This effect is achieved by the introduction of the routing path increment threshold $h_{\text {thres }}$.

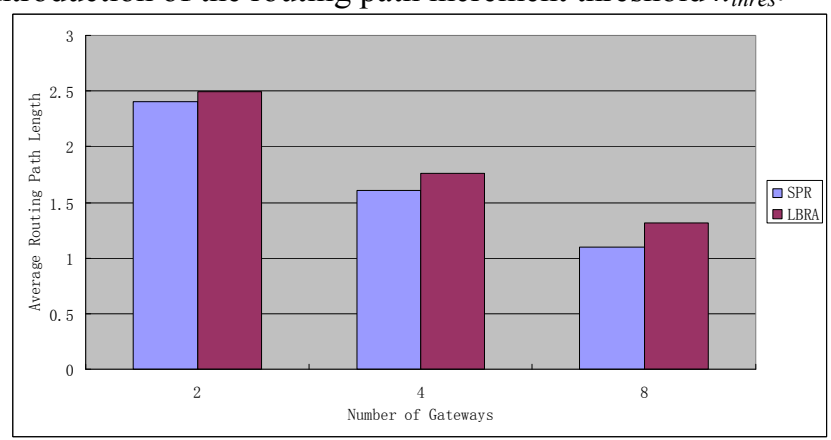

Figure 5. The impact of SPR and LBRA on average routing path length with different number of gateways.

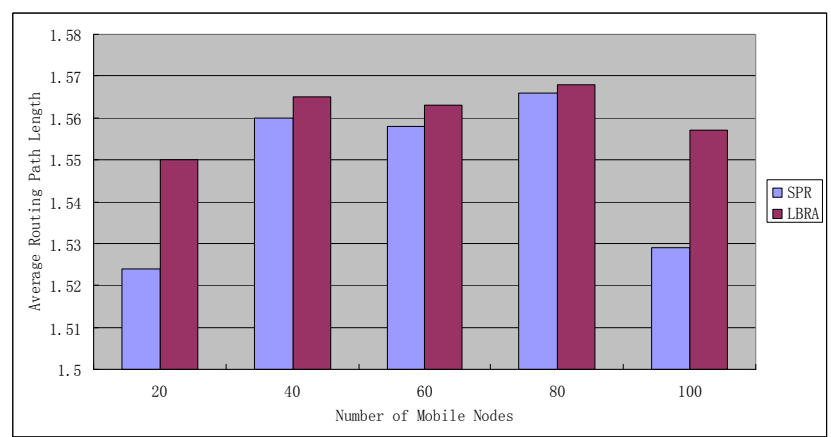

Figure 6. The impact of SPR and LBRA on average routing path length with different number of mobile nodes.

\section{Conclusions}

In this paper, we proposed a load balancing routing algorithm, called LBRA, to distribute the traffic load on gateways in MANET with Internet connectivity. To prevent the routing path becoming too long, a routing path increment threshold is introduced to restrict the routing path in a reasonably range. Through simulations, we showed the proposed algorithm LBRA can distribute the traffic load among multiple gateways uniformly and can restrict the increase of the routing path to, at most, $20 \%$ compared to SPR. In the future, we will investigate how to set the threshold dynamically to suit for different network scenarios.

\section{ACKNOWLEDGMENT}

This work is supported by the Defense Advance Research Project "Mobile Computing Network and Device Technology", China.

\section{REFERENCES}

[1] S. Corson and J. Macker, Mobile Ad hoc Networking (MANET): Routing Protocol Performance issues and Evaluation Considerations, Network Working Group, RFC2501, January 1999.

[2] Q. Le-Trung, P.E. Engelstad, T. Skeie and A. Taherkordi, LoadBalance of Intra/Inter-MANET Traffic over Multiple Internet Gateways, Proceedings of the 6th International Conference on Advances in Mobile Computing and Multimedia, Pages 50-57, 2008.

[3] Vinicius C. M. Borges, Erik Dimitrov, Marilia Curado and Edmundo Monteiro, RAILoB - A Routing Algorithm for Inter-cluster Load Balancing in Wireless Mesh Networks. The 9th Annual IEEE Consumer Communications and Networking Conference - Wireless Consumer Communication and Networking. pp: 904-909, Las Vegas, NV, USA, 2012.

[4] Choi, Hyoung-Gyu, SSeung-Jae Han, Load Balancing Routing for Wireless Mesh Networks: An Adaptive Partitioning Approach. 5th IEEE Consumer Communications and Networking Conference, Las Vegas, NV, USA 2008. pp:1-5

[5] Wen Song, Xuming Fang, Routing with Congestion Control and Load Balancing in Wireless Mesh Networks, the 6th International Conference on ITS Telecommunications Proceedings, 2006

[6] Chungui Liu, Yantai Shu, Lianfang Zhang, Jun Li, Efficient Multiple Gateways Load-Balancing and QoS Routing in Wireless Mesh Networks. 4th International Conference on Wireless Communications, Networking and Mobile Computing, Dalian, China. 2008 pp.1-4.

[7] J. Li, C. Blake, D. S. J. D. Couto, H. Lee and R. Morris, Capacity of Ad Hoc Wireless Networks, in Proc. ACM MobiCom '01, pp.61-69, July 2001.

[8] E. W. Dijkstra. A note on two problems in connection with graphs. Numerische Mathematik, 1(6):269-270, 1959.

[9] The Network Simulator - NS-2, http://www.isi.edu/nsnam/ns/

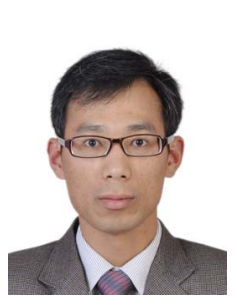

Yonghang Yan (Beijing, 1981) is a Ph.D. candidate in the School of Computer Science \& Technology, Beijing, China. He is working with Prof. Linlin $\mathrm{Ci}$ in the Mobile Computing and Network Technology Laboratory. His research interests include computer network, Mobile ad hoc network, wireless communication, mobile computing network and QoS.

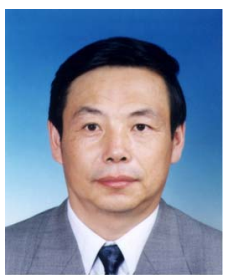

Linlin Ci (Beijing, 1950) is a professor in the School of Computer Science \& Technology, Beijing, China. $\mathrm{He}$ got Bachelor's degree in 1976 at Beijing Institute of Technology and the major is computer science and technology. He got Master's degree in 1986 at Northwestern Polytechnical University. His research focuses on mobile computing, Artificial Intelligence, wireless sensor network and DTN.

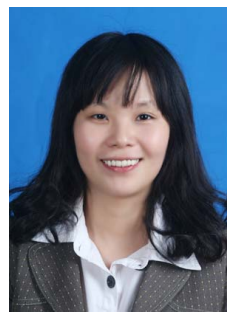

Ruiping Zhang (Zhengzhou, 1981) is an instructor at the school of computer science at Zhongyuan University of Technology. She graduated in Henan Normal University with a bachelor's degree majoring in computer science in July 2002. She achieved her Master's Degree in Wuhan University of Technology at May 2008. Her research interests contain computer network and mobile computing.

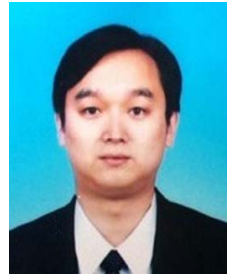

Zhiming Wang (Beijing, 1973) is a vice professor in the School of Computer Science \& Technology, Beijing Institute of Technology, China. His research interests focuses on mobile computing network and Artificial Intelligence and wireless communication. 